\title{
Experimental methods to study cultural differences in linguistics
}

Evangelia Adamou

CNRS

\section{Introduction and definitions}

A relatively recent development in linguistics is the use of experimental methods in different cultural settings. It takes place within a larger trend in psychology that questions the robustness of theories and generalizability of its findings following the observation that research predominantly relies on participants from so-called 'Western, educated, industrialized, rich, and democratic' (WEIRD) societies (Henrich et al. 2010). To set the scene for this discussion, let us start by looking more closely at the two keywords of this chapter: 'experimental' and 'cultural.'

'Experimentation' is a way for scientists to isolate, prepare, and manipulate their object of investigation and its environment in order to test and update a scientific theory, that is, a collection of laws and generalizations that aims to describe, explain, and predict a set of phenomena (for an overview of this topic in philosophy of science see Boyd and Bogen 2021). In comparison, 'observation' is when scientists note details about their object of investigation, either under natural or experimental conditions. Observation and experimentation may require the use of instruments and therefore the quality of measures obtained and their analysis is key to the success of the scientific endeavour. Since experiments are about control, the lab is the typical place to conduct them. A controlled environment may be crucial in physics, where scientists may also need to use experimental equipment that is only available in the lab (e.g., a particle accelerator). In addition, so-called 'field experiments,' conducted outside the lab, are common in many scientific disciplines like geochemistry and economics (e.g., where scientists test policy-related solutions in the real world). Similarly, linguistic experiments can be conducted in the field by slightly adjusting the experimental protocol (see sections 5 and 6 for more details).

Let us now turn to consider the deceptively intuitive notion of 'culture.' In a classic definition, the notion of culture encompasses those aspects of behaviour that are not genetically determined, but are acquired through social learning (Cavalli-Sforza and Feldman 1981). Culture is reflected in myths, legends, religion, body adornment, rules, daily routines, and use of production tools (Brown 2004). In addition, every society has different forms of social organization: it has rules for social groups, age grading, family, kinship systems, play, division of labour, exchange, cooperation, and reciprocity (Brown 2004). Contemporary anthropologists came to critically appraise the notion of culture itself, viewed as 'essentialized, localized, territorialized, bounded, highly shared, and symbolically coalesced' (Hirschfeld 2018: 233). Appadurai (1996) proposes instead a view of cultural spaces as fluid, heterogenous, fragmented, and constantly negotiated. Similarly, Claidiere and Sperber (2007) opt for the dynamic notion of 'cultural representations.' This means that researchers should not just aim to investigate differences between WEIRD and non-WEIRD populations but should also take into consideration diversity and variability within these populations (e.g., by including low-income, less-educated, and ethnically-diverse participants). 


\section{Historical perspectives}

The idea that cultural differences should take front stage in experimental psychology is not new. Boas (1930), a major figure in American anthropology, already criticizes the exclusive focus of psychologists on the individual, abstracting away from the social environment. Yet, Arnett (2008) find that $96 \%$ of participants in behavioural research publications from 2003-2007 were from WEIRD countries, although these represent roughly $12 \%$ of the world's population. Follow-up studies by Nielsen et al. (2017) and Rad et al. (2018) report no substantial change. In addition, Rad et al. (2018) note that participant samples are frequently unidentifiable and when they are, they rely on undergraduates and online samples with no information about participant characteristics such as socioeconomic status, education, and ethnicity.

Linguistics is equally concerned by these cultural biases. Psycholinguistics is a traditionally experimental subfield that studies how word meaning, sentence meaning, and discourse meaning are represented in the mind during speech production and comprehension. Anand et al. (2011) find that only 10 languages represent $85 \%$ of psycholinguistics publications and conferences, i.e., English, German, Japanese, French, Dutch, Spanish, Mandarin, Korean, Finnish, and Italian. In child language acquisition, a subfield that has a tradition of relying on both experimental and observational data, Kidd and Garcia (2021) arrive to a similar conclusion: in the past 45 years, only $1.5 \%$ of the world's 7000 languages is represented by at least one article ( $\mathrm{N}=103$ languages), with English and other well-studied Indo-European languages representing the great majority of publications. To my knowledge, there are no studies on the representation of different languages in the various subfields of experimental linguistics, like experimental phonology, semantics, pragmatics, syntax, and morphology (Hemforth 2013). Experimental phonetics, an interdisciplinary field of linguistics, physiology, and acoustics, most likely stands out for having the longest tradition of cross-linguistic research.

\section{Critical issues and topics}

Why is it important to study cultural differences in linguistics? First, we have to acknowledge the significance of the empirical database issue. There is considerable language diversity, with variety at every level, from linguistic sounds, to grammar, to meaning, and lexicon that still needs to be described (Evans and Levinson 2009). As a result, the search for linguistic universals has been repeatedly challenged by novel empirical evidence. For example, a proposed universal that was abandoned is 'baby talk,' the specific prosody that English, French, or German caregivers use to talk to infants. Cross-cultural and cross-linguistic studies demonstrate that baby talk is in fact a cultural convention (see Quiché Mayan in BernsteinRatner and Pye 1984). Another abandoned universal is the Possible Word Constraint, according to which a single consonant or cluster of consonants cannot form a word. El Aissati et al. (2012), however, found that this constraint does not apply in Tashelhit Berber, an Afroasiatic language that also allows clusters of consonants to be words. For more examples, see Evans and Levinson (2009) and Kidd and Garcia (2021).

The failure to find universals in such cultural and linguistic diversity should not come as a surprise if we admit that, although humans share a common biological basis, environment and experience determine which linguistic characteristics become conventionalized. Experiencedriven models, in particular, try to capture the complex and adaptive nature of language:

Language has a fundamentally social function. Processes of human interaction along with domain-general cognitive processes shape the structure and knowledge of language. Recent 
research in the cognitive sciences has demonstrated that patterns of use strongly affect how language is acquired, is used, and changes. These processes are not independent of one another but are facets of the same complex adaptive system (CAS). Language as a CAS involves the following key features: The system consists of multiple agents (the speakers in the speech community) interacting with one another. The system is adaptive; that is, speakers' behaviour is based on their past interactions, and current and past interactions to get her feed forward into future behaviour. A speaker's behaviour is the consequence of competing factors ranging from perceptual constraints to social motivations. The structures of language emerge from interrelated patterns of experience, social interaction, and cognitive mechanisms (Five Graces Group 2009: 1-2).

In sum, if we want to better understand how the interaction environment and linguistic experience shape languages, we have to move beyond student participant samples from the Global North and unidentifiable samples of participants recruited online.

\section{Current contributions and research}

This section offers an overview of some studies that have been successfully conducted with diverse populations and summarizes their findings.

\section{Experimental studies on language and cognition in diverse cultural settings}

A survey of experimental work to study cultural differences and their effects on language can only start with reference to the controversial 'linguistic relativity' or 'Sapir-Whorf hypothesis' (Whorf 1941/1956). The linguistic relativity hypothesis holds that our language habits shape the way we think of the real world. Nowadays, a weak version of the linguistic relativity hypothesis is largely accepted, acknowledging that language allows for faster performance and better discrimination of the real world and that it enhances memorization. The degree of detail in the linguistic categorizations in a given language reflects the cultural interests and communicative needs of its speakers.

Quantity. Frank et al. (2008) study cognition of quantity among the Amazonian Pirahã people who speak a language that does not have a special lexicon for number. The researchers performed two tasks, one of elicitation of numerals to confirm that the Pirahã do not use number language ( $\mathrm{N}=6$ participants), and one matching task with real life objects to test numerical cognition ( $\mathrm{N}=14$ participants). The study finds that, despite the absence of number words, speakers of Pirahã perceive and match exact quantities. The study also finds that lack of number words affects accuracy in memorization of large numbers of objects.

Colour. Gibson et al. (2017) worked with the Tsimane', a hunter-gatherer Amazonian population from Bolivia ( $\mathrm{N}=28-58$ participants in the various tasks). They conducted various colour naming tasks using chips and picture stimuli, as well as a memory colour task. They then compared the results to two control groups, a group of Bolivian Spanish speakers from the area ( $\mathrm{N}=20-25$ participants) and a group of English speakers in the US ( $\mathrm{N}=29-30$ participants). Analysis of the results suggests that the Tsimane' respond to their communicative needs for natural objects through an elaborate botanical vocabulary, not through colour names. However, the study also finds that introduction of coloured artefacts boosts the relevance of colour since their colour is not predictable. The number of colour names is therefore likely to increase in industrial societies when speakers are confronted to new communicational needs that require colour disambiguation. 
Space. A standard task to test memorization strategies of spatial relations in the field is 'Animals in a Row' (Levinson 2003). Participants in this task are asked to memorize the placement of three, small, toy animal figures positioned in a row in front of them. They are asked to recall this initial placement and reconstruct it a few meters away after a $180^{\circ}$ rotation. Overall, responses to the Animals in a Row task provide results confirming the linguistic relativity hypothesis, that is, that language habits predict the preferred memorization of spatial relations. However, Meakins et al. (2016) report that the Gurindji participants in Australia gave a majority of geocentric responses to the task (i.e., memorizing the animal figures with respect to a cardinal point) whether they spoke Gurindji, a Pama-Nyungan language with an elaborate system of cardinal terms ( $\mathrm{N}=30$ participants), or Gurindji Kriol, a mixed language resulting from an English-lexified creole and Gurindji that has not retained the Gurindji cardinal term system ( $\mathrm{N}=77$ participants).

Calderón et al. (2019) follow up on this observation by investigating an Indigenous population of Mexico, the Ngiguas. In the small rural community where the study was conducted, only some elders still speak Ngigua (Otomanguean), while the younger generations only speak Spanish. 17 Spanish monolinguals and 17 Ngigua-Spanish bilinguals responded to a localization task to allow for the study of both speech and co-speech gesture. Analysis of the results shows that Spanish monolinguals use geocentric co-speech gestures to support geocentric representations of space, as well as Spanish cardinal terms in an innovative manner. This confirms that transmission of traditional ways of representing space is possible in small, rural communities even when the Indigenous language is no longer spoken.

\section{Experimental studies among diverse bilingual populations}

Bilingualism is another subfield where the study of cultural differences is necessary to understand human language and cognition. Bilingualism is a dynamic and adaptive neurocognitive phenomenon across individuals and across the lifespan of a single individual (Pliatsikas 2019). It follows that if we only examine Western, middle-class, highly educated bilingual populations, we are missing out on the huge variety of bilingual experiences (Adamou 2021).

Language switching costs. For example, in the psycholinguistic literature, there is ample experimental evidence indicating slower reaction times when participants are asked to name pictures by alternating from one language to another. These are known as 'language switching costs' related to lexical access. However, researchers increasingly show that language switching costs can be reduced or even disappear when the experimental tasks are closer to participants' real-life experience with codeswitching (see Gullifer et al. 2013 for a study with SpanishEnglish bilinguals from the US).

In line with this work, Adamou and Shen (2019) conducted the first experimental study of sentence processing in a typologically rare form of codeswitching used by Romani (Indic)Turkish (Altaic) simultaneous bilinguals from Greece. This Romani-Turkish variety shows an interesting split in morphology: Turkish nouns inflect in Romani for case, number, and gender, like other Romani nouns do, but Turkish verbs systematically combine with the Turkish person, tense-aspect-modality morphology, and valency morphemes, contrasting with Romani verbs that combine with Romani verbal morphology. In summary, the findings from a picturematching and a word monitoring experiment show that Romani-Turkish bilinguals anticipate codeswitching based on prior experience ( $\mathrm{N}=37$ and 49 participants). More specifically, when the input aligns with their expectations, there are no processing costs. This study paves the way to experimental studies with speakers of the few fully-fledged mixed languages that have been identified in the world. Mixed languages result from the systematic combination of two 
languages and arise under specific social circumstances where a community of speakers forges a mixed cultural identity.

Priming. 'Priming' refers to the observation that processing one stimulus (the prime) unconsciously affects the processing of a subsequent stimulus (the target). This mechanism is well-documented in language, including for 'structural priming' where the activation of one structure affects the production and the comprehension of a subsequent structure. A metaanalytic study also shows that structural priming operates among monolinguals and to a lesser extent among bilinguals (Mahowald et al. 2016). In theory, cross-language structural priming among bilinguals could be a mechanism that drives contact-induced change in the long run (Loebell and Bock 2003). However, such ongoing change is not easily observed in languages like English, with a strong written tradition and with prescriptive norms that are strengthened by formal education.

Kootstra and Şahin (2018) are the first to experimentally demonstrate the relevance of crosslanguage priming in structural change in a bilingual population speaking Papiamento, a Spanish and Portuguese-based Creole, and Dutch (Germanic). The researchers tested priming in dative structures among Papiamento-Dutch bilinguals living in the Netherlands ( $\mathrm{N}=37$ participants) and in Aruba ( $\mathrm{N}=25$ participants). They found that Papiamento speakers who are in daily contact with Dutch (in the Netherlands) exhibit higher levels of priming by Dutch dative structures than those who have less intense contact with Dutch (in Aruba).

Similarly, Adamou et al. (2021) tested the role of cross-language priming in adjective (ADJ)-noun (N) order among 90 bilinguals of Romani (Indic) and Romanian (Romance) from Romania. The study reveals significant cross-language priming effects, whereby bilinguals favour the use of N-ADJ order in Romani immediately following a N-ADJ sentence read in Romanian whereas the inherited ADJ-N Romani order only benefits from priming when speakers read a Romani sentence with an ADJ-N order.

\section{Experimental studies on typologically rare linguistic phenomena in diverse cultural settings}

Typologically rare linguistic phenomena offer a unique opportunity to test theoretical claims and expand our knowledge of what is possible in human language.

Relative clauses. In a classic article, Keenan and Comrie (1977) propose that, crosslinguistically, subjects are more accessible to relativization than direct objects, indirect objects, and oblique objects; this preference is known as the Accessibility Hierarchy. Since then, there has been a large body of work with typologically diverse languages confirming the original explanation that the Accessibility Hierarchy 'directly reflects the psychological ease of comprehension' (Keenan and Comrie 1977: 88).

More recently, a series of experimental studies was conducted in languages that use fully ambiguous relative clauses (RCs). The rationale is that these ambiguous RCs can offer an unconfounded result for universal subject preference and an experiment has the advantage of neutralizing any disambiguating semantic and pragmatic cues that can be otherwise found in natural conversations.

The first experimental study tested subject preference in two ergative Mayan languages, Ch'ol and Q'anjob'al, using a picture-matching comprehension experiment (Clemens et al. 2015). 63 Ch'ol and 100 Q'anjob'al speakers participated in the study in Mexico and Guatemala respectively. The results for the ambiguous RCs in both languages confirm universal subject preference favouring an ergative-subject interpretation (68\% in Ch'ol and 74\% in Q'anjob'al). The study also shows that participants were faster in the subject responses than in the object ones offering support to the ease of comprehension explanation. 
Another experimental study tested subject preference in ambiguous RCs for both postnominal and prenominal RCs in Chamorro, an Austronesian language (Borja et al. 2016). Results from 135 participants show object preference in prenominal RCs, but subject preference for postnominal RCs. The study also finds a subject processing advantage in both prenominal and postnominal RCs.

Adamou (2017) adapted this experiment for Ixcatec, a critically-endangered Otomanguean language of Mexico with less than ten speakers. The analysis shows that $63 \%$ of the Ixcatec ambiguous RCs are interpreted as subject $\mathrm{RCs}$ ( $\mathrm{N}=7$ participants). Results from reaction times show that subject $\mathrm{RC}$ interpretations are numerically faster than object $\mathrm{RC}$ interpretations, although this difference does not reach significance. This lack of significance may be due to the small size of the sample or it could suggest that Ixcatec comprehenders do not go through an initial stage of subject interpretation before proceeding to an object interpretation in agreement with spoken corpus preferences where transitive subject RCs are as frequent as object RCs.

Nominal tense. Nominal tense is a linguistic phenomenon that has recently been reported for a small number of languages across the world to refer to the use of grammatical morphology on argument nominals with an independent temporal interpretation from that of the clause (Nordlinger and Sadler 2004). This is a highly controversial phenomenon in that it challenges our view of nouns as time stable and, in particular, our experience with languages that encode tense only through verbal but not nominal morphology.

Descriptive work identified that deictic suffixes carry temporal information on their own in Pomak, a non-standardized Slavic variety spoken in Greece. But, in practice, what does it mean for speakers to have nominal tense in their language? In Adamou and Haendler (2020), an experimental study using a response-time experiment with 40 Pomak participants demonstrates for the first time that speakers of a language with nominal tense can decide whether a noun phrase is past or future without any additional information from an adverb or a verb and in the absence of pragmatic and semantic cues. Beyond the relevance of the results for Pomak, this study introduces an experimental method that can easily be used to test the existence of nominal tense in other languages.

\section{Main research methods}

Experiments are quantitative methods. Typically, in order to obtain sufficient statistical power, the number of participants and experimental trials is high. However, Navarro-Torres et al. (2021) stress that rich characterization of the sample may be as or even more important than a poorly characterized large sample. For example, when considering the impact of bilingualism on cognition, a binary characterization 'bilingual' vs. 'monolingual' is not informative enough since we know that fine-grained distinctions in age of acquisition, proficiency, daily use, and frequency of codeswitching are moderating factors.

In an experiment, scientists identify a 'dependent variable,' something that is the focus of the study, and test whether it is affected by an 'independent variable.' For example, the dependent variable can be the reaction times of participants in a response task and the independent variable the position of the button on the right or left. At present, complex statistical models allow for the examination of more variables as well as their interactions.

Standard experimental techniques include the use of 'control' or 'comparison' groups that differ on the variable that is hypothesized to be the main cause for an observed behaviour (e.g., when studying bilingual populations, speaking language $\mathrm{A}$ and $\mathrm{B}$, it is common to include a monolingual control group who only speaks language $\mathrm{A}$ and then compare responses in 
language A for the two groups); randomization of trials, so that the order of appearance of the stimuli would not influence the results; inclusion of stimuli as 'fillers,' that is, items that the experimenter is not interested in and that are used in order to prevent participants from identifying the goal of the study (note that researchers sometimes analyse responses to fillers to see if the participants performed the task as expected); and 'norming' (i.e., rating) of the stimuli prior to the experiment by non-participants to make sure they are as well-formed as the experimenter thought they would be.

Some experimental designs that have been successfully conducted in the field are judgement tasks, picture-matching tasks, priming tasks, and several semi-experimental tasks, including picture naming and speech production tasks.

\section{Judgment tasks}

Acceptability judgment tasks are a reliable tool in linguistic research (Sprouse et al. 2013), in particular when working with a large sample of participants (Gibson and Fedorenko 2013). Participants in these tasks judge the acceptability or naturalness of linguistic stimuli using a five-point or a seven-point scale (small numbers are for negative judgments and high numbers for positive judgments). To facilitate the memorization of the scale for participants who have little experience with computers or this kind of formal testing, it is possible to use stickers on the keyboard: a smiley face on the key for the best score and a frown for the worst. To make sure participants understand the task and are allowed to negatively evaluate some stimuli, practice sentences can serve as anchors for the highest and lowest points in the scale with feedback by the experimenter prior to the experiment. Moreover, instead of asking the participants whether the stimuli are 'correct' or 'acceptable,' experimenters can ask whether they are 'natural' in the community (e.g., Adamou and Haendler 2020).

\section{Picture-matching tasks}

Picture-matching experiments are inspired by the visual world paradigm which was developed by Dahan and Tanenhaus (2004) for eye tracking. In picture-matching experiments, participants view a pair of pictures and are asked to match one of the pictures with the content of a spoken sentence that they hear. The pictures can be drawings (e.g., Clemens et al. 2015; Borja et al. 2016), pictures found on the Internet (e.g., Adamou and Shen 2019), or photographs of people, objects, and situations that the community is familiar with and that are specifically taken for the study (e.g., Adamou 2017; Calderón et al. 2019). The task is generally presented on a laptop. However, to make sure that the elderly speakers of Ixcatec (in their 80s) would be comfortable enough to perform the task, Adamou (2017) presented the stimuli printed in A4 size. The stimuli were manually randomized through reshuffling in the beginning of each session. The sessions were filmed and reaction times were measured from the onset of the audio stimuli to the moment where participants pointed to the picture. Later, Calderón et al. (2019) conducted this experiment with elderly Ngigua speakers and found that it can also work well on a laptop. Lack of familiarity with a computer does not seem to be an impediment as long as the instructions are clear (also see Adamou and Shen 2019 for a similar experience).

\section{Priming tasks}

A typical design for a priming production experiment consists of a sentence trial and a picture trial. Participants first read or listen to a sentence (sentence trial) and then describe a picture (picture trial). A priming effect is observed when the linguistic structure chosen in the picture trial follows the structure of the sentence trial (e.g., Kootstra and Şahin 2018; Adamou et al. 2021). 
Word monitoring is a different kind of priming experiment. This is a task where participants listen to a sentence and are asked to press a button as soon as they hear a specific word (target). The time it takes to press the button for the target provides information about the ease of processing of the preceding word (prime). To make sure that participants are not just searching for the target word without processing the sentence, a comprehension question can follow each trial (e.g., Adamou and Shen 2019).

\section{Semi-experimental tasks}

In addition to the experimental tasks listed above, there are a number of semi-experimental tasks that can be useful. For example, verbal fluency tasks allow researchers to assess a bilingual speaker's language dominance at the moment of the study. In the semantic version of the task, speakers are asked to produce in one minute as many words as possible belonging to a specific semantic category (e.g., body parts, animals, fruits and vegetables) in one language and then in the other (see Calderón et al. 2019). The language in which the greatest number of words is produced, is the dominant language at the moment of the study. In the phonological version of the task, speakers need to produce as many words as possible beginning with specific letters or sounds. However, this version should not be used with participants with low literacy levels as it is known to be a good indicator of illiteracy (in alphabetic scripts) and is modulated by the level of education (Petersson et al. 2000).

Other popular semi-experimental tasks are naming tasks in which speakers are asked to name real life objects or objects depicted in drawings or illustrated in pictures, for example, to study color names (Gibson et al. 2017). In an information structure phonetic study in Ixcatec, real-life objects were shown in a first session (e.g., fruits); photographs of these objects were shown in a second session. This combination allowed for enough repetitions while avoiding a habituation process that could interfere with information structure status (Adamou et al. 2018).

More generally, speech production tasks are semi-experimental. For example, to investigate spatial language and cognition, participants were asked to provide a description of the localization of two buildings while being filmed (Calderón et al. 2019). This allows to analyse both speech and co-speech gesture for all participants while controlling their position with respect to cardinal points in order to differentiate between spatial representations that are geocentric (based on cardinal points) and egocentric (based on the speaker's viewpoint).

\section{Recommendations for practice}

This section offers a brief practical guide to build and conduct an experiment in diverse cultural and linguistic settings following eight steps:

\section{Step 1: Identify a research question}

The point of departure in an experimental study should always be the identification and formulation of a clear research question. For researchers who are community outsiders, familiarity with the community and the language under study are essential to this endeavour so as to not directly transpose research questions from well-described languages and populations to lesser-described ones, but to identify the most relevant research questions. In line with the community-based research frame, researchers should recruit research assistants from the community who will actively participate in the elaboration of the experimental design as well as its implementation. Appropriate credit and compensation are expected, including offering co-authorship in scientific papers. Reminder: as in any workplace, maintaining respectful and 
ethical professional relationships in the field is paramount. When applicable, it is possible to integrate the research study in a local association's activities and negotiate its goals with the association's representatives and members. Researchers who are members of the community are also concerned by the ways to best communicate and prepare their research albeit in a different way (Cruz Cruz 2020).

\section{Step 2. Clearly formulate your research question and predictions}

In experimental work, it is important to start by formulating clear research questions and predictions. This is always done before conducting the experiments. Preregistrations of the experimental protocol, including information about the hypothesis, the data that will be collected, and their analysis are possible using the Open Science Framework (OSF) (https://osf.io/).

\section{Step 3. Choose your experimental design}

A great number of experimental paradigms are available in the literature. In practice, some leeway is allowed in adapting these designs to the field (see section 5 for some examples).

Most research institutions require that research protocols be approved by research ethics committees before implementation. In particular, research involving vulnerable groups such as minorities and groups who are economically disadvantaged is treated with particular caution. The Statement of Ethics of the American Anthropological Association is a good starting point for a general reflection on ethical issues (see http://ethics.americananthro.org/ethics-statement0-preamble/), but also see Pérez González (2021) for an Indigenous academic perspective and Gaby and Woods (2020) for a discussion of how linguists should relate to Indigenous peoples and their languages.

\section{Step 4. Prepare your stimuli and build your experiment}

In psycholinguistic experiments, many tasks are built around written stimuli as they present several advantages (e.g., no confound by differences in prosody; better control of the length of the stimuli). However, aural stimuli are better adapted to the study of less-described languages as these are rarely written and taught at school. When recording the stimuli, it is important to make sure not to introduce artefacts due to dialectal differences, and therefore work with speakers from within the community. A good idea is to conduct a 'norming study' prior to the experiment. In a norming study, participants not involved in the experiment rate the various stimuli on a five or seven-point scale for well-formedness (as in a judgment task; see above). Researchers should eliminate the stimuli that receive a mean rating below a given threshold (e.g., three in a five-point scale). Length of the stimuli should be controlled for to ensure comparability and eventually allow for calculating reaction times. Fillers need to be used to distract the attention of participants from the main research question. Finally, stimuli should be randomized for each participant. Regarding visual stimuli, it is best to use coloured photographs rather than black and white line drawings given that little-educated participants have difficulties recognizing the latter (Reis et al. 2006). One can either use existing visual stimuli or create their own, culturally-adapted stimuli (see Borja et al. 2016; Adamou 2017; Adamou et al. 2018; Calderón et al. 2019). To build an experiment, a free open source experiment builder is Open Sesame (Mathôt et al. 2012). 


\section{Step 5. Select your participants}

Before conducting experiments in the field, it is important to have had the opportunity to conduct extensive participant observation to comprehend the sociolinguistic background and decide who could take part in the study (e.g., age range, languages spoken, etc.). At the beginning of the experimental session, experimenters can conduct a short sociolinguistic interview to assess education, literacy, dialectal variation, and multilingualism among the participants.

Prior to their participation in the experiment, participants are asked to sign a consent form or simply record their approval. This is when researchers can explain what participants will be asked to do during the experiment; that they can leave before the end of the experiment if they feel uncomfortable; why the researchers are conducting this study; what are the benefits for the community; whether participants will receive compensation and who funds the research; where the collected data will be stored and published; how the researchers will ensure respect for the privacy and confidentiality of participants' personal information. In addition to individual consent, researchers often need to obtain community consent through the appropriate institutions (e.g., the community's general assembly) as well as national and/or local authorities.

In psycholinguistic research, it is standard practice to compensate participants for their time, but in the field, it is important to first understand cultural norms with regard to financial compensation. Also keep in mind that it is not ethically acceptable to exercise pressure or allure potential low-income participants by exclusively offering financial incentives. Alternatives to individual financial compensation may be a small gift to participants or a financial contribution to a local association.

\section{Step 6. Collect your data}

Always allow extra time for data collection in the field as participants may be busy with everyday life tasks and not be available for the experimental study as planned. Even if you cannot conduct the experiment in the lab, try to conduct it in a similar location for all the participants: a quiet room in the house of participants, a classroom at the local school, a room in a collective structure that is accessible to all the participants.

Unlike standard practice in most lab research, where the presence of the researcher during the experiments is not required, in the field it is best that the researcher be present in order to make sure that everything goes according to plan. Moreover, the researchers who will conduct the experiment in the field must be familiar with the computer programme (e.g. Open Sesame) that they will use in order to check at the beginning of every session if it is recording the data properly and adjust as needed.

\section{Step 7. Analyse your data}

Experimental linguists typically learn to use statistical methods for data analysis. This is an important investment in time and constant training is needed to follow developments in the field of statistics. Alternatively, collaborating with a researcher who is familiar with statistics is an excellent way to ensure state-of-the-art analyses.

\section{Step 8. Interpret your results}

Once the statistical analyses are available, researchers can confront the results with the predictions formulated at the beginning of the research project. 


\section{Future directions}

Experimental linguistics is a fast-growing field. But will cross-linguistic and cross-cultural representation follow this development?

\section{Challenges}

There are several challenges to the use of experimental methods outside the lab. First, practical reasons are at play. As with any research conducted in the field, impediments include accessibility to remote locations, access to electricity, and adverse climate conditions (Hellwig 2019). Other practical reasons may include difficulties to transfer high-quality portable equipment to the field. However, these problems are increasingly being solved thanks to recent technological progress allowing the use of laptops, cameras, recorders, and mobile eye-trackers even in isolated places. In addition, with travel restrictions due to the Covid-19 pandemic (starting in 2020), many researchers turned to web-based research, a special type of field research that allows reaching large and heterogeneous populations using smartphones, personal computers, and the Internet (Dufau et al. 2011; Boase and Humphreys 2018). Prior experience shows that response times are accurately measured when they are recorded locally and then shared via the web (Enochson and Culbertson 2015).

Second, linguists who work in diverse linguistic and cultural settings, and language communities themselves, may have different research priorities. For example, when studying an undescribed language, the primary goal is to produce a grammatical sketch and a dictionary. When working on an endangered language, the priority is language documentation and revitalization. Experimental work can therefore be introduced once primary goals are met. Experimental methods are not necessarily antagonistic with these goals since they allow researchers to work with a great number of participants in a given community, whether these are speakers of the language under study or not (in the latter case they can collaborate as part of a control or comparison group) (Adamou 2021).

Finally, a major hurdle is that experiments are designed for Western, highly educated participants and that there is still little knowhow to design experiments that can work for socially and culturally diverse populations. For example, Sauerland (2018) shares his experience with the Amazonian Pirahã community. During a weeklong stay, he recruited 16 speakers to respond to a truth value judgment task that aimed to test syntactic recursion following a heated debate about whether Pirahã exhibited recursion or not. Pirahã participants were asked to listen to the pre-recorded statements of a Pirahã speaker, e.g., 'I have been to the moon,' reported accurately or inaccurately by a second Pirahã speaker, e.g., 'X said 'I have been to the sun.' Participants in the experiment had to decide whether the second speaker heard well or said the truth. The experiment did not work as expected and the author admits that one reason might be that the speakers who recorded the utterances were well-respected senior members of the community and that it may have been culturally and socially inappropriate for the participants in the study to challenge what the elders said by qualifying their statements as 'false.' Another interesting example of both a failed and a successful experiment is related in Mulak et al. (2021). A team of researchers, including researchers who were familiar with the community, conducted a word learning experiment with 34 Nungon speakers from Papua New Guinea. Whereas the first experiment yielded null results, the second experiment was successful after adjusting the instructions to make them clearer, reducing the cognitive load of the task, and framing the study within a real-world context. 


\section{Ways forward}

As a community, we can support diversity in experimental linguistics by explicitly encouraging the submission of papers from under-described languages in conferences and following through on the publication of this research in international journals. As individual researchers, we can change our practices, even when we do not work in a cross-cultural perspective, to include a thoughtful characterization of our sample and carefully discuss its potential for generalization.

Last but certainly not least, we have to keep in mind that cross-cultural and cross-linguistic experimental research raises specific ethical issues. Whereas researchers in most lab-based experimental research are not familiar with their participants, familiarity with the communities that participate in experimental work in the field should be the starting point. Otherwise we run the risk of turning experimental research in the field into an extractive kind of science. As in other research fields, intense collaboration with the various communities and reciprocity should be the guiding principles in this endeavour.

\section{Further Reading}

Adamou, E., 2021. The Adaptive Bilingual Mind: Insights from Endangered Languages, Cambridge: Cambridge University Press.

This book demonstrates how experimental psycholinguistic methods can be successfully applied outside the lab, including with speakers of endangered languages. In addition to the quantitative, experimental results, the book offers ample qualitative linguistic and ethnographic observations.

\section{Gillioz, C. and Zufferey, S., 2020. Introduction to Experimental Linguistics. John Wiley \& Sons.}

This is an accessible and detailed introduction to experimentation in linguistics, from concepts to methods and data analyses. It draws on examples from the various fields of linguistics.

Majid, A., 2021. 'Olfactory language requires an integrative and interdisciplinary approach', Trends in Cognitive Sciences. https://doi.org/10.1016/j.tics.2021.03.004

Majid takes the example of olfactory language to argue that not only do we need crosscultural and cross-linguistic experimental research, but that we also need to adopt a transdisciplinary approach that includes linguistic elicitation, text analysis, conversation analysis, ethnographic observation, case studies, surveys, and computational approaches, among others.

Meakins, F., Green, J. and Turpin, M. 2018. Understanding Linguistic Fieldwork, London: Routledge.

This is an up-to-date student's guide to linguistic fieldwork presenting a variety of methods. The volume discusses examples from colonial and postcolonial settings, including Australia, the United States of America, Canada, South America, and Africa.

Rappaport, J., 2020. Cowards don't make history: Orlando Fals Borda and the origins of participatory action research, Durham, NC: Duke University Press.

This is a historical overview of Participatory Action Research methods focusing on the collaboration between the sociologist Orlando Fals Borda with Indigenous and peasant populations in Colombia in the early 1970s. It should help researchers reflect on what it means to conduct research in the field. 


\section{Related topics}

Experimental methods to study child language, bilinguals, older populations, language and thought

\section{References}

Adamou, E. (2017) 'Subject preference in Ixcatec relative clauses (Otomanguean, Mexico)', Studies in Language 41: 872-913. https://doi.org/10.1075/s1.16055.ada

Adamou, E. (2021) The Adaptive Bilingual Mind: Insights from Endangered Languages, Cambridge: Cambridge University Press.

Adamou E., Feltgen, Q. and Padure, C. (2021) 'A unified approach to the study of language contact: Cross-language priming and change in adjective/noun order', International Journal of Bilingualism online first https://doi.org/10.1177/13670069211033909

Adamou, E., Gordon, M. and Gries, S. T. (2018) 'Prosodic and morphological focus marking in Ixcatec (Otomanguean)' in E. Adamou, K. Haude and M. Vanhove (eds) Information Structure in Lesser-Described Languages: Studies in Prosody and Syntax. Amsterdam and Philadelphia: John Benjamins. https://doi.org/10.1075/slcs.199.03ada

Adamou, E. and Haendler, Y. (2020) 'An experimental approach to nominal tense: Evidence from Pomak (Slavic)', Language 96(3): 507-50.

Adamou, E. and Shen, X. R. (2019) 'There are no language switching costs when codeswitching is frequent', International Journal of Bilingualism 23: 53-70. https://doi.org/10.1177/1367006917709094

Anand, P., Chung, S. and Wagers, M. (2011) 'Widening the net: Challenges for gathering linguistic data in the digital age', National Science Foundation SBE 2020 planning activity. https://people.ucsc.edu/ mwagers/papers/WideningtheNet.AnandChungWagers.pdf (Accessed 24 September 2021).

Appadurai, A. (1996) Modernity at Large: Cultural Dimensions of Globalization, Minneapolis: University of Minnesota Press.

Arnett, J. J. (2008) 'The neglected 95\%: why American psychology needs to become less American', The American Psychologist 63(7): 602-14.

Bernstein-Ratner, N. and Pye, C. (1984) 'Higher pitch in BT is not universal: acoustic evidence from Quiché Mayan', Journal of Child Language 11: 515-22. doi.org/10.1017/S0305000900005924

Boas, F. (1930). 'Some problems of methodology in social sciences', in L. D. White (ed.) The New Social Science, Chicago: University of Chicago Press.

Boase, J. and Humphreys, L. (2018) 'Mobile methods: Explorations, innovations, and reflections', Mobile Media \& Communication 6(2): 15362. https://doi.org/10.1177/2050157918764215

Borja, M. F., Chung, S. and Wagers, M. (2016) 'Constituent order and parser control processes in Chamorro', in A. Camp, Y. Otsuka, C. Stabile and N. Tanaka (eds), Proceedings of the 21st Annual Meeting of the Austronesian Formal Linguistics Association, Canberra: AsiaPacific Linguistics.

Boyd, N. M. and Bogen, J. (2021) 'Theory and observation in science', in E. N. Zalta (ed.) The Stanford Encyclopedia of Philosophy, https://plato.stanford.edu/archives/fall2021/entries/science-theory-observation/.

Brown, D. E. (2004) 'Human universals, human nature and human culture', Daedalus 133: 4754. https://doi.org/10.1162/0011526042365645

Calderón, E., De Pascale, S. and Adamou, E. (2019) "How to speak "geocentric" in an "egocentric" language: A multimodal study among Ngigua-Spanish bilinguals and Spanish 
monolinguals in a rural community of Mexico', Language Sciences 74: 24-46. https://doi.org/10.1016/j.langsci.2019.04.001

Cavalli-Sforza, L. L. and Feldman, M. W. (1981) Cultural Transmission and Evolution: A Quantitative Approach, Princeton: Princeton University Press.

Claidiere, N. and Sperber, D. (2007) 'The role of attraction in cultural evolution', Journal of Cognition and Culture 7: 89-111.

Clemens, L. E., Coon, J., Mateo Pedro, P., Morgan, A. M., Polinsky, M., Tandet, G. and Wagers, M. (2015) 'Ergativity and the complexity of extraction: A view from Mayan', Natural Language and Linguistic Theory 33(2): 417-69.

Cruz Cruz, E. (ed.) (2020) 'Theoretical reflections around the role of fieldwork in linguistics and linguistic anthropology: Contributions of Indigenous researchers from southern Mexico', Language Documentation and Conservation special publication 22-23. http://nflrc.hawaii.edu/ldc/sp23/

Dahan, D. and Tanenhaus, M. K. (2004), 'Continuous mapping from sound to meaning in spoken-language comprehension: immediate effects of verb-based thematic constraints', Journal of Experimental Psychology. Learning, Memory, and Cognition 30: 498-513. https://doi.org/10.1037/0278-7393.30.2.498

Dufau, S., Duñabeitia, J. A., Moret-Tatay, C., McGonigal, A., Peeters, D., Alario, F.-X., et al. (2011) 'Smart phone, smart science: How the use of smartphones can revolutionize research in cognitive science', PLoS ONE 6(9): e24974. https://doi.org/10.1371/journal.pone.0024974

El Aissati A., McQueen J. M. and Cutler, A. (2012) 'Finding words in a language that allows words without vowels', Cognition 124: 79-84.

Enochson, K. and Culbertson, J. (2015) 'Collecting psycholinguistic response time data using Amazon Mechanical Turk', PLoS ONE 10(3): e0116946.

Evans, N. and Levinson, S. C. (2009) 'The myth of language universals: Language diversity and its importance for cognitive science', Behavioral and Brain Sciences 32: 429-48. https://doi.org/10.1017/s0140525x0999094x

Five Graces Group, Beckner, C., Blythe, R., Bybee, J., Christiansen, M. H., Croft, W., Ellis, N. C., Holland, J., Ke, J., Larsen-Freeman, D. and Schoenemann, T. (2009) 'Language is a complex adaptive system: Position paper', Language Learning 59: 1-26. https://doi.org/10.1111/j.1467-9922.2009.00533.x

Frank, M. C., Everett, D. L., Fedorenko, E. and Gibson, E. (2008) 'Number as a cognitive technology: Evidence from Pirahã language and cognition', Cognition 108: 819-24. https://doi.org/10.1016/j.cognition.2008.04.007

Gaby, A. and Woods, L. (2020) 'Toward linguistic justice for Indigenous people: A response to Charity Hudley, Mallinson, and Bucholtz', Language 96(4): e26880. doi:10.1353/lan.2020.0078.

Gibson, E. and Fedorenko, E. (2013) 'The need for quantitative methods in syntax and semantics research', Language and Cognitive Processes 28: 88-124. https://doi.org/10.1080/01690965.2010.515080

Gibson, E., Futrell, R., Jara-Ettinger, J., Mahowald, K., Bergen, L., Ratnasingam, S., Gibson, M., Piantadosi, S. T. and Conway, B. R. (2017) 'Color naming across languages reflects color use', Proceedings of the National Academy of Sciences of the United States of America 114(40): 10785-90. https://doi.org/10.1073/pnas.1619666114

Gullifer, J., Kroll, J. F. and Dussias, P. E. (2013) 'When language switching has no apparent cost: Lexical access in sentence context', Frontiers in Psychology 4: 1-13. 
Hellwig, B. (2019) 'Linguistic diversity, language documentation and psycholinguistics: The role of stimuli', Language Documentation and Conservation 16. http://hdl.handle.net/10125/24855

Hemforth, B. (2013) 'Experimental linguistics', in Oxford Bibliographies Online in Linguistics. doi: 10.1093/obo/9780199772810-0112.

Henrich, J., Heine, S. and Norenzayan, A. (2010) 'The weirdest people in the world?', Behavioral and Brain 6ciences 33(2-3): doi:10.1017/S0140525X0999152X

Hirschfeld, L. (2018) 'The Rutherford atom of culture', Journal of Cognition and Culture 18: 231-61.

Kidd, E. and Garcia, R. (2021) 'How diverse is child language acquisition?' https://doi.org/10.31234/osf.io/jpeyq

Kootstra, G. J. and Şahin, H. (2018) 'Crosslinguistic structural priming as a mechanism of contact-induced language change: Evidence from Papiamento-Dutch bilinguals in Aruba and the Netherlands', Language 94: 902-30. https://doi.org/10.1353/lan.2018.0050

Levinson, S. C. (2003) Space in Language and Cognition, Cambridge: Cambridge University Press.

Loebell, H. and Bock, K. (2003) 'Structural priming across languages', Linguistics 41: 791824. https://doi.org/10.1515/ling.2003.026

Mahowald, K., James, A., Futrell, R. and Gibson, E. (2016) 'A meta-analysis of syntactic priming in language production', Journal of Memory and Language 91: 5-27. https://doi.org/10.1016/j.jml.2016.03.009

Mathôt, S., Schreij, D. and Theeuwes, J. (2012) 'OpenSesame: An open-source, graphical experiment builder for the social sciences', Behavior Research Methods 44: 314-324. https://doi.org/10.3758/s13428-011-0168-7

Meakins, F., Jones, C. and Algy, C. (2016) 'Bilingualism, language shift and the corresponding expansion of spatial cognitive systems', Language Sciences 54: 1-13. https://doi.org/10.1016/j.langsci.2015.06.002

Mulak, K. E., Sarvasy, H. S., Tuninetti, A. and Escudero, P. (2021) 'Word learning in the field: Adapting a laboratory-based task for testing in remote Papua New Guinea', PLoS ONE 16: e0257393. https://doi.org/10.1371/journal.pone.0257393

Navarro-Torres, C. A. Beatty-Martínez, A. L., Kroll, J. F. and Green, D. W. (2021) 'Research on bilingualism as discovery science', Brain and Language 222: 105014.

Nielsen, M., Haun, D., Kärtner, J. and Legare, C. H. (2017) 'The persistent sampling bias in developmental psychology: A call to action', Journal of Experimental Child Psychology 162: 31-8. https://doi.org/10.1016/j.jecp.2017.04.017.

Nordlinger, R. and Sadler, L. (2004) 'Nominal tense in a crosslinguistic perspective', Language 80: 776-806.

Pérez González, J. (2021), 'The ethical principles of linguistic field work methodologiesAccording to whom?' [Translated from Spanish] in E. Cruz Cruz (ed.) Theoretical Reflections Around the Role of Fieldwork in Linguistics and Linguistic Anthropology: Contributions of Indigenous Researchers from Southern Mexico. Language Documentation \& Conservation: 131-52. http://hdl.handle.net/10125/24988

Petersson, K. M., Reis, A., Askelöf, S., Castro-Caldas, A. and Ingvar, M. (2000) 'Language processing modulated by literacy: a network analysis of verbal repetition in literate and illiterate subjects', Journal of Cognitive Neuroscience 12: 364-382. https://doi.org/10.1162/089892900562147 
Pliatsikas, C. (2019) 'Understanding structural plasticity in the bilingual brain: The dynamic restructuring model', Bilingualism: Language and Cognition 6: 1-13. https://doi.org/10.1017/S1366728919000130

Rad, M. S., Martingano, A. J. and Ginges, J. (2018) 'Toward a psychology of Homo sapiens: Making psychological science more representative of the human population', Proceedings of the National Academy of Sciences 115(45): 1140111405. https://doi.org/10.1073/pnas.1721165115

Reis, A., Faísca, L., Ingvar, M. and Petersson, K. M. (2006) 'Color makes a difference: Twodimensional object naming in literate and illiterate subjects', Brain and Cognition 60: 4954. https://doi.org/10.1016/j.bandc.2005.09.012

Sauerland, U. (2018) 'False Speech Reports in Pirahã: A Comprehension Experiment', in L. Amaral, M. Maia, A. Nevins, and T. Roeper (eds) Recursion Across Domains, Cambridge: Cambridge University Press.

Sprouse, J., Schütze, C. T. and Almeida, D. (2013) 'A comparison of informal and formal acceptability judgments using a random sample from Linguistic Inquiry 2001-2010', Lingua 134: 219-248. https://doi.org/10.1016/j.lingua.2013.07.002

Whorf, B. L. (1941) 'The relation of habitual thought and behavior to language', in L. Spier (ed.) Language, Culture, and Personality: Essays in Memory of Edward Sapir. Menasha: Sapir Memorial Publication Fund. Reprinted in J. B. Carroll (ed.) Language, Thought, and Reality: Selected Writings of Benjamin Lee Whorf. New York and London: MIT Press and John Wiley \& Sons. 